\title{
Dynamical properties in the space of fuzzy numbers
}

\author{
Jiř́i Kupka ${ }^{1}$ \\ ${ }^{1}$ Institute for Research and Applications of Fuzzy Modeling, University of Ostrava, Czech Republic
}

\begin{abstract}
This paper is a contribution to the theoretical foundations of the theory of fuzzy dynamical systems. More precisely, we study relations between a given discrete dynamical system on the space $X$ and its fuzzy counterpart - Zadeh's extension defined on the space of fuzzy sets on $X$. We provide a short discussion and a brief survey of some recent results devoted to various (especially chaotic) dynamical properties, a special attention is paid to fuzzifications on the space of fuzzy numbers, i.e., to fuzzy sets with connected $\alpha$-cuts.
\end{abstract}

Keywords: Zadeh's extension, fuzzy dynamical system, fuzzification, transitivity, chaotic properties, topological entropy

\section{Introduction}

In this paper by $\left(X, d_{X}\right)$ we denote a compact metric space with the metric $d_{X}$. A discrete dynamical system is considered to be a pair $(X, \varphi)$. For other notions and notations mentioned in this section, we rather refer to Section 2. It is known ([21]) that any given (crisp) discrete dynamical system $(X, \varphi)$ uniquely induces a dynamical system $(\mathbb{F}(X), \tilde{\varphi})$ that is defined on the space $\mathbb{F}(X)$ of all fuzzy compact subsets of $X$. The map $\tilde{\varphi}$ is usually called fuzzification (or Zadeh's extension) (see (3)).

The current situation is the following - shortly speaking, fuzzy dynamical systems defined in this way are often used in various applications (see e.g., [20], [5] and included references) and several methods for their effective simulations have been invented (e.g., [8]) until now. However, our knowledge concerning dynamical behavior of such fuzzy discrete dynamical systems is rather poor despite of the fact that the classic topological dynamics (the theory of dynamical systems) has been developed for decades. Our current problem is to study and clarify relations between the original dynamical system $(X, \varphi)$ and its induced fuzzy counterpart. There are several papers devoted to this question - for example, we can mention [9], [17], [18], [6] and [15] where various dynamical properties of fuzzy discrete dynamical systems were studied in general.

However the problem is still far from being solved for instance, from the practical point of view it is reasonable to pay our attention especially to the space of fuzzy numbers (i.e., fuzzy sets with connected $\alpha$-cuts) and not to answer only generally stated questions. The aim of this paper is to provide a brief survey of some recent results and also a short discussion on which dynamical properties can be useful to study (especially in the space of fuzzy numbers). This contribution also contains some new results - the most interesting results (namely, Theorems 3 and 4) claim that the set-valued extension of any crisp discrete dynamical system is a dynamical factors of the fuzzy dynamical system induced by the same dynamical system. As consequences of mentioned theorems various recently established results can be easily obtained.

The structure of this paper is the following - several basic notions are introduced mainly in Section 2. Then we discuss the density of the set of periodic points in Subsection 3.1, we continue with various dynamical properties related to the transitivity in Subsection 3.2 and, at the end, we discuss the size of topological entropy in various spaces of fuzzy sets in Subsection 3.3. Finally, it is common to finish with some concluding remarks (see Section 4).

\section{Preliminaries}

Let $\left(X, d_{X}\right), \mathbb{N}$ and $I$ be a compact metric space, the set of integers and the interval $[0,1]$, respectively. As usually, $\operatorname{int}(A), \bar{A}$ denote the interior and topological closure of a given set $A \subseteq X$.

For a discrete dynamical system $(X, \varphi)$ and a given point $x \in X$, we establish basic notions from topological dynamics. The $n$-th iteration of the point $x$ is defined inductively by

$$
\varphi^{0}(x)=x \text { and } \varphi^{n+1}(x)=\varphi\left(\varphi^{n}(x)\right)
$$

for any $n \in \mathbb{N}$. Then, the sequence $\left\{\varphi^{n}(x)\right\}_{n \in \mathbb{N}}$ of all iterations of $x$ is called the trajectory and points of this trajectory form a set called orbit of the point $x$. Sometimes we distinguish between forward and backward trajectory, resp. orbit. Limit points of considered trajectories are called $\omega$-limit points of the point $x$, and the union $\omega_{\varphi}(x)$ of all $\omega$-limit points of the point $x$ is the $\omega$-limit set of the point $x$ with respect to the map $\varphi$. Iterations, trajectory and other notions of a given set $A \subseteq X$ can be defined analogously.

Simpler $\omega$-limit sets can have special names - we say that the point $x \in X$ is fixed if $\varphi(x)=x$ or periodic if $\varphi^{k}(x)=x$ for some $k \in \mathbb{N}$. Then the minimal $k$ satisfying this condition is called a period of $x$. Moreover, $\omega(\varphi)$ and $P(\varphi)$ denote the set of $\omega$-limit and periodic points of the map $\varphi$, respectively.

Consider now two discrete dynamical systems $(X, \varphi)$ and $(Y, \psi)$. If there exists a continuous surjective map $h: X \rightarrow Y$ such that $h \circ \varphi=\psi \circ h$, the map $h$ is called a semiconjugacy, $(X, \varphi)$ and $(Y, \psi)$ are semiconjugated and $(Y, \psi)$ is a dynamical factor of $(X, \varphi)$, respectively. If such a map $h$ is bijective, it is called conjugacy and 
we say that $(X, \varphi)$ and $(Y, \psi)$ are conjugated. It is well known (e.g., [3]) that numerous dynamical properties (e.g., the size of topological entropy) are preserved by conjugacies, resp., are inherited by semi-conjugacies.

\subsection{Chaotic Properties}

A given continuous map $\varphi: X \rightarrow X$ (notation $\varphi \in$ $C(X)$ ) is called transitive if for any non-empty open subsets $U, V \subseteq X$, there exists an integer $k$ such that $k$-th iteration of $U$ intersects $V$. The map $\varphi$ is totally transitive if the each iteration $\varphi^{n}$ of $\varphi$ is transitive, $n \in \mathbb{N}$. The map $\varphi$ is weakly mixing if the product map $\varphi \times \varphi: X \times X \rightarrow X \times X$ is transitive. The map $\varphi$ is called strongly mixing (resp., shortly mixing) if for any non-empty open subsets $U, V \subseteq X$ there exists a threshold $m \in \mathbb{N}$ for which $\varphi^{k}(U) \cap V \neq \emptyset$ for $k \geq m$. The map $\varphi$ is topologically exact (or simply exact or locally eventually onto) if for any non-empty subset $U \subseteq X$, some iteration of $\varphi$ covers the whole space $X$.

The following implications are currently known among the properties mentioned above:

$$
\text { exactness } \Rightarrow \text { strong mixing } \Rightarrow
$$

weak mixing $\Rightarrow$ total transitivity $\Rightarrow$ transitivity.

We say that a map $\varphi: X \rightarrow X$ is Devaney chaotic if it is transitive and has dense set of periodic points in $X$. If $X$ is infinite, these two conditions ensures (for details see [4] and [10]) that the map $\varphi$ depends sensitively on initial conditions. There are also other variants of Devaney chaos (namely, exact Devaney chaos and total Devaney chaos) that are defined by replacing the notion of transitivity by exactness or total transitivity, respectively, in the definition of Devaney chaos.

\subsection{Topological entropy}

Now we introduce the definition of topological entropy. It is a commonly accepted tool (resp. number) representing the dynamical complexity of $\varphi$ (or the dynamical system $(X, \varphi))$. Let $K \subseteq X$ be compact and fix $\varepsilon>0$ and $n \in \mathbb{N}$. Then a set $E \subseteq K$ is $(n, \varepsilon, K, \varphi)$-separated (by the map $\varphi$ ) if for any $x, y \in E, x \neq y$, there exists $k \in\{0,1, \ldots, n-1\}$ satisfying $d_{X}\left(\varphi^{k}(x), \varphi^{k}(y)\right)>\varepsilon$. The cardinality of any maximal $(n, \varepsilon, K, \varphi)$-separated set in $K$ is usually denoted by $s_{n}(\varepsilon, K, \varphi)$. We can put

$$
s(\varepsilon, K, \varphi)=\limsup _{n \rightarrow \infty} \frac{1}{n} \log s_{n}(\varepsilon, K, \varphi) .
$$

It is known that $s(\varepsilon, K, \varphi)$ increases when $\varepsilon$ decreases. Now the topological entropy of $\varphi$ is

$$
h(\varphi)=\sup _{K} \lim _{\varepsilon \rightarrow 0} s(\varepsilon, K, \varphi) .
$$

If the space $X$ is compact, then

$$
h(\varphi)=\lim _{\varepsilon \rightarrow 0} s(\varepsilon ., X, \varphi),
$$

However, if $X$ is not compact, we use the following definition ([7]) of topological entropy (see also [6] for further details):

$$
\operatorname{ent}(\varphi)=\sup \left\{h\left(\left.\varphi\right|_{K}\right): K \in \mathcal{K}_{i n(\varphi)}(X)\right\},
$$

where $\mathcal{K}_{i n(\varphi)}(X)$ denotes the set of $\varphi$-invariant (i.e., $\varphi(A) \subseteq A$ for each $A \subseteq X$ ) compact subsets of $X$.

\subsection{Spaces of fuzzy Sets}

Let us introduce spaces of fuzzy sets we consider in this contribution and their topological structures. For nonempty closed subsets $A, B$ of $X$, the Hausdorff metric between $A$ and $B$ is defined, as usually (e.g. [12]), by

$D_{X}(A, B)=\inf \left\{\varepsilon>0 \mid A \subseteq U_{\varepsilon}(B)\right.$ and $\left.B \subseteq U_{\varepsilon}(A)\right\}$, where $U_{\varepsilon}(A)$ denotes an open $\varepsilon$-neighborhood of the set A.

Later we work with the space $\mathbb{K}(X)$ of all nonempty compact subsets of $X$ equipped with the Hausdorff metric $D_{X}$ and also with its topological subspace $\mathbb{K}_{c}(X)$ consisting of nonempty connected closed subsets of $X$. It is well known (e.g., [16]) that the mentioned spaces $\left(\mathbb{K}(X), D_{X}\right)$ and $\left(\mathbb{K}_{c}(X), D_{X}\right)$ are compact, complete and separable for $X$ possessing the same three properties.

Now we would like to introduce some spaces of fuzzy sets. Formally, a fuzzy set $A$ on the space $X$ is a function $A: X \rightarrow I$. The $\alpha$-cuts (or the $\alpha$-level sets) $[A]_{\alpha}$ and the support $\operatorname{supp}(A)$ of a given fuzzy set $A$ are defined as usually -

$$
[A]_{\alpha}=\{x \in X \mid A(x) \geq \alpha\}, \text { for } \alpha \in[0,1],
$$

and

$$
\operatorname{supp}(A)=\overline{\{x \in X \mid A(x)>0\} .}
$$

Within this contribution we consider the system $\mathbb{F}(X)$ of all upper semi-continuous fuzzy sets $A$ on $X$. Moreover, for $\alpha \in(0,1]$, put

$$
\mathbb{F}^{\alpha}(X)=\left\{A \in \mathbb{F}(X) \mid \max _{x \in X}\{A(x)\}=\alpha\right\}
$$

and let $\mathbb{F}^{1}(X)$ denote the system of normal fuzzy sets on $X$. The space $\mathbb{F}_{c}(X)$ of so-called fuzzy points (or fuzzy numbers) consisting of $A \in \mathbb{F}(X)$ for which each nonempty $\alpha$-cut is connected in $X$ is also studied. Finally, by an empty fuzzy set $\emptyset_{X}$ we call a map $\emptyset_{X}(x)=0$ for each $x \in X, \mathbb{F}_{0}(X)$ denotes the system of all nonempty fuzzy sets and $\mathbb{F}_{c}^{\alpha}(X):=\mathbb{F}^{\alpha}(X) \cap \mathbb{F}_{c}(X)$ for $\alpha \in(0,1]$.

The following metric is usually taken on the space of nonempty fuzzy sets. A levelwise metric $d_{\infty}$ on $\mathbb{F}_{0}(X)$ is given by

$$
d_{\infty}(A, B)=\sup _{\alpha \in(0,1]} D_{X}\left([A]_{\alpha},[B]_{\alpha}\right) .
$$

This equality defines the levelwise metric correctly for non-empty fuzzy sets $A, B \in \mathbb{F}_{0}(X)$ with identical maximal values, since the Hausdorff distance $D_{X}$ can 
be measured only between two non-empty closed subsets of the space $X$. However there is a relatively easy way to cope with this problem ([13]).

It is known that considered spaces of fuzzy sets $\left(\mathbb{F}(X), \mathbb{F}^{1}(X), \mathbb{F}_{c}^{1}(X)\right.$ and $\left.\mathbb{F}_{c}(X)\right)$ equipped with the levelwise topology, i.e. the metric topology induced by $d_{\infty}$, are complete but are not compact and are not separable (see [13] and references therein) in general. It should be noticed that the metric $d_{\infty}$ is one of the three most commonly used metrics in fuzzy topological dynamics and the levelwise topology induced by $d_{\infty}$ is stronger than the others (denoted by $d_{E}$ and $d_{S}$ ) for more details we refer again to [13] and references therein. From this point of view it is natural to study various dynamical properties in the levelwise topology since many properties can be easily shifted to the remaining topological structures.

\subsection{Zadeh's and Set-valued Extensions}

In this subsection we specify which extensions can be induced by a given discrete dynamical system $(X, \varphi)$. Its set-valued extension is a self-map $\bar{\varphi}$ on $\mathbb{K}(X)$ defined by the expression

$$
\bar{\varphi}(A)=\varphi(A)
$$

for any $A \in \mathbb{K}(X)$. It is well known that $\bar{\varphi}$ is continuous when the space $\mathbb{K}(X)$ is endowed with the metric topology (called Vietoris topology) induced by the Hausdorff metric $D_{X}$. We also consider its restriction $\bar{\varphi}_{c}:=\left.\bar{\varphi}\right|_{\mathbb{K}_{c}(X)}$ to the space of closed connected subsets of $X$.

However, $\varphi \in C(X)$ can be naturally extended to the space of fuzzy sets on $X$. Namely, a fuzzification (or Zadeh's extension) of the dynamical system $(X, \varphi)$ is a map $\tilde{\varphi}: \mathbb{F}(X) \rightarrow \mathbb{F}(X)$ defined by the expression

$$
(\tilde{\varphi}(A))(x)=\sup _{y \in \varphi^{-1}(x)}\{A(y)\}
$$

for arbitrary $A \in \mathbb{F}(X)$ and $x \in X$.

Some time ago the continuity of the fuzzification $\tilde{\varphi}: \mathbb{F}(X) \rightarrow \mathbb{F}(X)$ was proved (see [18]) provided $\varphi: X \rightarrow X$ is continuous too. The last statement was recently generalized about the case of locally compact metric spaces in [13]. Finally, it is obvious that the continuity is preserved for any restriction of $\tilde{\varphi}$, for instance, for $\tilde{\varphi}_{c}:=\left.\tilde{\varphi}\right|_{\mathbb{F}_{c}(X)}$ defined on the space of fuzzy numbers.

It is known ([9]) that the original map (resp. its setvalued extension) and its fuzzification are related via $\alpha$ cuts, i.e. for any $\alpha \in(0,1]$ and any $A \in \mathbb{F}(X)$,

$$
\varphi\left([A]_{\alpha}\right)=[\tilde{\varphi}(A)]_{\alpha} .
$$

Similarly also the equality $\varphi(\operatorname{supp}(A))=\operatorname{supp}(\tilde{\varphi}(A))$ can be proven.

Thus, we may distinguish several discrete dynamical systems - namely, the original (crisp) one $(X, \varphi)$, the set-valued (induced) ones $(\mathbb{K}(X), \bar{\varphi}),\left(\mathbb{K}_{c}(X), \bar{\varphi}\right)$ and the fuzzy (fuzzified) ones $(\mathbb{F}(X), \tilde{\varphi}),\left(\mathbb{F}_{c}^{1}(X), \tilde{\varphi}_{c}\right)$ etc. It is known ([13]) that the original dynamical system is a factor of none of remaining dynamical systems we consider here. However in this paper we show that set-valued extensions are dynamical factors of relevant fuzzified ones.

We conclude this subsection with a simple observation.

Lemma 1 Any dynamical system $(X, \varphi)$ can be embedded to $(\mathbb{K}(X), \bar{\varphi}), \quad(\mathbb{K}(X), \bar{\varphi}), \quad\left(\mathbb{F}^{1}(X), \tilde{\varphi}\right)$, $\left(\mathbb{F}_{c}^{1}(X), \tilde{\varphi}\right)$ etc. The set-valued extension $(\mathbb{K}(X), \bar{\varphi})$ (resp., $(\mathbb{K}(X), \bar{\varphi})$ ) can be embedded to $\left(\mathbb{F}^{1}(X), \tilde{\varphi}\right)$, $\left(\mathbb{F}_{c}^{1}(X), \tilde{\varphi}\right)$.

Proof. Consider a map $x \mapsto\{x\}$ and characteristic functions $x \mapsto \chi_{\{x\}}, A \mapsto \chi_{A}$ for $x \in X$ and $A \subseteq X$, respectively.

\subsection{Symbolic dynamics}

To construct some subsequent examples we need some notions from symbolic dynamics. Namely, if we fix $n \in$ $\mathbb{N}, \Sigma_{n}=\{0,1,2, \ldots, n-1\}^{\mathbb{N}}$ denotes the space of all one-sided sequences of $n$ symbols and $x_{i}$ denotes $i$-th coordinate of $x \in \Sigma_{n}$ for $i \in \mathbb{N}$, i.e. $x=x_{1} x_{2} x_{3} \ldots$. The metric $\delta_{n}$ on $\Sigma_{n}$ is defined as follows - for any two sequences $x^{1}, x^{2} \in \Sigma_{n}$

$$
\delta_{n}\left(x^{1}, x^{2}\right)=\sum_{m \geq 1} \frac{v\left(x_{m}^{1}, x_{m}^{2}\right)}{n^{m}},
$$

where

$$
v\left(x_{m}^{1}, x_{m}^{2}\right)= \begin{cases}1 & x_{m}^{1} \neq x_{m}^{2} \\ 0 & \text { otherwise }\end{cases}
$$

It is known that the metric space $\left(\Sigma_{n}, \delta_{n}\right)$ is also compact and separable. Let

$$
\left[x_{1}, x_{2}, \ldots, x_{k}\right]
$$

denote the set of sequences $z \in \Sigma_{n}$ for which $z_{i}=$ $x_{i}$ for $i \in\{1,2, \ldots, k\}$. Finally, we define an adding machine $\tau: \Sigma_{2} \rightarrow \Sigma_{2}$ as usually by

$$
\tau(x)=x+1000 \ldots, \text { for any } x \in \Sigma_{2},
$$

where the addition in this expression is in the base two and from the left to the right. For instance, $\tau(0001001 \ldots)=1001001 \ldots, \tau(111010 \ldots)=$ $000110 \ldots$ etc.

Finally, a shift map $\sigma_{n}: \Sigma_{n} \rightarrow \Sigma_{n}$ is defined by $\sigma_{n}\left(x_{1} x_{2} x_{3} x_{4} \ldots\right)=x_{2} x_{3} x_{4} \ldots$. It is well known that this map has various specific dynamical properties, e.g., $\sigma_{n}$ is exact or $P\left(\sigma_{n}\right)$ is dense in $\Sigma_{n}$ and so on.

\section{Main Results}

Let us shortly summarize recent progress in the task established at the beginning of this paper. We would like to mention [13] where some basic facts on fuzzifications have been explained. Then, probably the first paper where relations among chaotic properties (Li-Yorke 
chaos, $\omega$-chaos etc.) that are mutually inherited between the original system $(X, \varphi)$ and its fuzzy counterpart $\left(\mathbb{F}^{1}(X), \Phi\right)$ was [15]. However, three versions of Devaney chaos were not studied therein. Consequently, the same problem devoted to Devaney chaotic fuzzy dynamical systems was solved in [14] recently. In the rest of this section we recall some of those results and we discuss analogous results for fuzzifications defined on the space of fuzzy numbers. First we restrict our attention to notions related to Devaney chaotic maps - namely to the periodic density and transitivity.

\subsection{Periodic Density}

In this section we provide results concerning density of the set of periodic points. We show via piecewise constant fuzzy sets that periodic densities of the set-valued and fuzzy extension of the original system coincide.

We say that an upper semi-continuous $\operatorname{map} \varphi$ is piecewise constant if and only if there is a finite number of sets $D_{i} \subseteq X$ such that $\bigcup \bar{D}_{i}=X$ and $\varphi$ is constant on $D_{i}$ except for finitely many points. Piecewise constant fuzzy sets has the following property.

Lemma 2 ([14]) For any $B \in \mathbb{F}(X)$ and $\varepsilon>0$ there exists a piecewise constant $A \in \mathbb{F}(X)$ such that $d_{\infty}(A, B)<\varepsilon$, i.e., the set of piecewise constant fuzzy sets is dense in $\mathbb{F}(X)$.

For completeness, since $\max A=\max B$ for each pair $A, B \in U$, it is possible to show that the set of piecewise constant fuzzy sets is dense also in each $\mathbb{F}^{\beta}(X)$ for each $\beta \in(0,1]$.

It was proved recently that if $\varphi \in C(X)$ has dense set of periodic points then both $\bar{\varphi}$ and $\tilde{\varphi}$ have the same property (see [18] and [19]). The converse implications are not valid - this fact can be demonstrated on any adding machine.

It is well known that any set $A \subseteq \mathbb{K}\left(\Sigma_{2}\right)$ of the form (5) is closed and open. It is clear that $\tau^{2^{k}}(x)$ and $x$ coincide on the first $k$ elements, i.e. if $\tau^{2^{k}}(x)=$ $s_{1} s_{2} \ldots s_{k} \ldots$ and $x=t_{1} t_{2} \ldots t_{k} \ldots$ then $s_{i}=t_{i}$ for each $i=1,2, \ldots, k$. Consequently, any set $A \subseteq \mathbb{K}\left(\Sigma_{2}\right)$ of the form (5) is periodic with respect to the map $\bar{\tau}$ with period $2^{k}$. We can easily show even more, namely that the set of periodic points of $\bar{\tau}$ is dense in $\mathbb{K}\left(\Sigma_{2}\right)$.

Lemma 3 ([14]) $P(\bar{\tau})$ is dense in $\mathbb{K}\left(\Sigma_{2}\right)$.

This result shows that an adding machine serves as a counterexample to (6) since we are able to prove the following relationship between $\bar{\varphi}$ and $\tilde{\varphi}$.

Theorem 1 ([14]) Let $\varphi \in C(X)$. Then $\tilde{\varphi}$ is periodically dense in $\mathbb{F}(X)$ (resp. $\mathbb{F}^{\alpha}(X)$ for any $\left.\alpha \in(0,1]\right)$ if and only if $\bar{\varphi}$ is periodically dense in $\mathbb{K}(X)$.

For completeness (see e.g., [2]), we recall that any adding machine represents an infinite minimal set, i.e. nonempty closed invariant set such that no proper subset has the same three properties. Obviously no infinite minimal set contains a periodic point. Consequently, it follows from Theorem 1 that

$$
P(\tilde{\varphi}) \text { dense } \nRightarrow P(\varphi) \text { dense. }
$$

Similar result we obtain for the fuzzification restricted to the space of fuzzy numbers. Really, since the space $\Sigma_{2}$ is totally disconnected, $\mathbb{K}_{c}\left(\Sigma_{2}\right)$ consists of singleton sets, i.e. $\mathbb{K}_{c}\left(\Sigma_{2}\right)=\Sigma_{2}$. By the minimality of $\Sigma_{2},\left.\bar{\varphi}\right|_{\mathbb{K}_{c}\left(\Sigma_{2}\right)}$ has no periodic point. On the other hand, Lemma 3 claims that $P(\bar{\tau})$ is dense in $\mathbb{K}\left(\Sigma_{2}\right)$, i.e.

$$
P\left(\left.\bar{\varphi}\right|_{\mathbb{K}_{c}\left(\Sigma_{2}\right)}\right) \text { dense } \nRightarrow P(\bar{\varphi}) \text { dense. }
$$

By using similar argument we can easily see that when the initial space is totally disconnected, then the condition

$$
P\left(\left.\bar{\varphi}\right|_{\mathbb{K}_{c}(X)}\right) \text { dense } \Leftrightarrow P(\bar{\varphi}) \text { dense. }
$$

Similar argument can be used for

$$
P\left(\left.\tilde{\varphi}\right|_{\mathbb{F}_{c}(X)}\right) \text { dense } \Leftrightarrow P(\tilde{\varphi}) \text { dense. }
$$

whenever the initial space $X$ is totally disconnected. On the other hand, when $X$ is connected then this condition can be easily violated even in one-dimensional spaces. To demonstrate this we present the following example.

Example 1 Consider an interval map $f: I \rightarrow I$ known as a tent map a defined by

$$
f(x)= \begin{cases}2 x & x \in[0,1 / 2] \\ 2-2 x & \text { orherwise }\end{cases}
$$

It is easy to see that, for any nonempty open $U \subseteq I$, $\bar{U} \neq I$, we have

$$
\operatorname{diam}(U)<\operatorname{diam}(f(U)),
$$

and

$$
U \subseteq f^{n}(U)
$$

for some $n \in \mathbb{N}$.

Recall that $U \subseteq f(U)$ ensures a fixed point in $U$. Then the second condition implies the existence of a periodic point of period $n$ in $U$ (hence $P(f)$ is dense in I) and the first condition implies that no nondegenerated interval distinct from $I$ can be periodic (hence $P\left(\bar{f}_{c}\right)$ is not dense in I).

Remark 1 Similar arguments can be used to demonstrate that $f$ from Example 1 is transitive while $\bar{f}_{c}$ is not.

There exists a relatively easy formal justification of the phenomenon demonstrated in Example 1 that is valid for all interval maps. Namely, for $f \in C(I)$ with dense set of periodic points, we have just two possibilities (see e.g., [2]) - either each point is periodic or $P(f)$ is not closed. The first case obviously tends to the fact that either each point is fixed or there exists the unique fixed 
point and remaining point are periodic points of period 2 . In both cases, $P\left(\bar{f}_{c}\right), P\left(\tilde{f}_{c}\right)$ are also closed. This case is not demonstrated by the tent map.

Further, the second case $(P(f)$ is dense but not closed) occurs only for interval maps possessing socalled basic set, i.e., maximal $\omega$-limit set containing periodic points. Basic sets are $\omega$-limit sets that are covered by periodic trajectory of some periodic interval $J$ of period $p$ such that $\left.f^{p}\right|_{J}$ is mixing. This property immediately implies that no proper nondegenerated subinterval of $J$ can be periodic.

\subsection{Transitivity and related properties}

In fact, we knew only a few results about transitive fuzzy dynamical systems so far. Recently, [18] H. RománFlores and his colleague proved that the transitivity of the fuzzification $\tilde{\varphi}$ implies the transitivity of the original $\operatorname{map} \varphi$. They also showed by using an irrational rotation on the unit circle as the crisp dynamical system that the converse implication is not valid. It can be easily seen that (see [15]), by using (4), no fuzzification can be transitive on the whole space $\mathbb{F}(X)$ of fuzzy sets.

Consequently, it was necessary to restrict our attention to more reasonable subspaces of fuzzy sets - for instance, to the space $\mathbb{F}^{1}(X)$ of normal fuzzy sets. Recently we proved that fuzzification on the space $\mathbb{F}^{1}(X)$ of normal fuzzy sets can be transitive.

Theorem 2 ([14]) The space of normal fuzzy sets equipped by the topology induced by the levelwise metric admits a transitive fuzzification.

Remark. To prove this theorem we used a shift map $\sigma_{n}$ and the fact that $\sigma_{n}$ is mixing. It seems that this assumption cannot be weakened by weak or mild mixing, for instance. It is not sufficient the original dynamical system to be only transitive or totally transitive - then any irrational rotation on the unit circle serves as the counterexample.

According to the previous remark, the following relationship between the original map $\varphi$ and its fuzzification can be proved.

Proposition 1 ([14]) If the original map $\varphi \in C(X)$ is mixing then $\tilde{\varphi}$ is transitive on $\mathbb{F}^{1}(X)$.

And we also proved that the transitivity of the fuzzified system implies that the original system is also transitive.

Lemma 4 ([14]) Let $(X, \varphi)$ be a discrete dynamical system. If its fuzzification $\tilde{\varphi}$ is transitive on $\left(\mathbb{F}^{1}(X), d_{\infty}\right)$ then the map $\varphi$ has the same property.

In the remaining part of this section let us concern mainly to the space $\mathbb{F}_{c}^{1}(X)$ of fuzzy numbers on $X$. We can follow the approach that was used in [18] or [15] for investigating relations between $\varphi$ and its fuzzification.
It can be shown that there exists an operator connecting topological structures of the set-valued and fuzzified dynamical systems. Namely, for any $U \subseteq \mathbb{K}(X)$ we define

$$
\vartheta(U):=\left\{A \in \mathbb{F}^{1}(X) \mid \operatorname{supp}(A) \in U\right\} .
$$

For instance, the following properties of this operator were proved.

Lemma 5 ([14]) For any $U, V \subseteq \mathbb{K}(X)$ and $\varphi \in C(X)$ we have

(i) $\vartheta(U) \neq \emptyset$ if and only if $U \neq \emptyset$,

(ii) a subset $U$ is a non-empty open subset of $X$ if and only if $\vartheta(U)$ is a non-empty open subset of $\mathbb{F}^{1}(X)$,

(iii) $\vartheta(U \cap V)=\vartheta(U) \cap \vartheta(V)$,

(iv) $\tilde{\varphi}(\vartheta(U)) \subseteq \vartheta(\bar{\varphi}(U))$.

Remark. We explained in [14] that properties (i)-(iv) in the last lemma can be proved for the space of fuzzy points $\mathbb{F}_{c}^{1}(X)$. In this case an operator

$$
\vartheta_{c}(U):=\left\{A \in \mathbb{F}_{c}^{1}(X) \mid \operatorname{supp}(A) \in U\right\} .
$$

has to be considered instead of $\vartheta$.

By using the operator $\vartheta$ several propositions can be deduced, e.g., see the following results and look in [18].

Proposition 2 ([14]) If $\tilde{\varphi}$ is transitive on $\left(\mathbb{F}^{1}(X), d_{\infty}\right)$ then $\bar{\varphi}$ is transitive on $\mathbb{K}(X)$.

Proposition 3 ([14]) If $\tilde{\varphi}$ is totally transitive on $\left(\mathbb{F}^{1}(X), d_{\infty}\right)$ then $\bar{\varphi}$ is totally transitive on $\mathbb{K}(X)$.

Proposition 4 ([14]) If $\tilde{\varphi}$ is exact on $\left(\mathbb{F}^{1}(X), d_{\infty}\right)$ then $\bar{\varphi}$ is exact on $\mathbb{K}(X)$.

With respect to the remark following Lemma 5, technical proofs of the mentioned propositions can be applied also to normal fuzzy sets with connected $\alpha$-cuts. Therefore we immediately obtain the following statements.

Proposition 5 ([14]) If $\tilde{\varphi}$ is transitive on $\left(\mathbb{F}_{c}^{1}(X), d_{\infty}\right)$ then $\bar{\varphi}$ is transitive on $\mathbb{K}_{c}(X)$.

Proposition 6 ([14]) If $\tilde{\varphi}$ is totally transitive on $\left(\mathbb{F}_{c}^{1}(X), d_{\infty}\right)$ then $\bar{\varphi}$ is totally transitive on $\mathbb{K}_{c}(X)$.

Proposition 7 ([14]) If $\tilde{\varphi}$ is exact on $\left(\mathbb{F}_{c}^{1}(X), d_{\infty}\right)$ then $\bar{\varphi}$ is exact on $\mathbb{K}_{c}(X)$. 
Now we would like to present that there exists another (simpler and more general) way how to prove results of that kind. We easily prove that set-valued discrete dynamical system is a dynamical factor of the fuzzy one. This allows us immediately to obtain numerous results explaining relations between these dynamical systems.

Theorem 3 For any dynamical system $(X, \varphi)$, there exists a semiconjugacy $h: \mathbb{F}^{1}(X) \rightarrow \mathbb{K}(X)$.

Proof. Consider a dynamical system $(X, \varphi)$ and its extensions $(\mathbb{K}(X), \bar{\varphi})$ and $\left(\mathbb{F}^{1}(X), \tilde{\varphi}\right)$. For any $\alpha \in(0,1]$, put

$$
E_{\alpha}(U):=\left\{A \in \mathbb{F}^{1}(X) \mid[A]_{\alpha}=U\right\}
$$

and

$$
E_{0}(U):=\left\{A \in \mathbb{F}^{1}(X) \mid \operatorname{supp}(A)=U\right\} .
$$

Now let $\alpha^{\prime} \in I$ be fixed and a map $h_{\alpha^{\prime}}: \mathbb{F}^{1}(X) \rightarrow$ $\mathbb{K}(X)$ be defined as follows:

$$
h_{\alpha^{\prime}}(A)=U \text { for which } A \in E_{\alpha^{\prime}}(U) .
$$

It is easy to see that any $A \in \mathbb{F}^{1}(X)$ belongs to the unique set $E_{\alpha^{\prime}}$, i.e., $h_{\alpha^{\prime}}$ is well defined. Further, for any $U \in \mathbb{K}(X)$ we have $E_{\alpha^{\prime}}(U) \neq \emptyset$, i.e., $h_{\alpha^{\prime}}$ is surjective. If any sequence $\left\{A_{n}\right\}_{n \in \mathbb{N}} \subseteq \mathbb{F}^{1}(X)$ converges to $A_{0} \in \mathbb{F}^{1}(X)$ in the levelwise topology, the definition of $d_{\infty}$ directly implies the uniform convergence of $\left\{\left[A_{n}\right]_{\alpha}\right\}_{n \in \mathbb{N}}$ to $\left[A_{0}\right]_{\alpha}$ in all $\alpha$-cuts, i.e., $h_{\alpha^{\prime}}$ is continuous.

To finish this proof it suffices to show $h_{\alpha^{\prime}} \circ \tilde{\varphi}=\bar{\varphi} \circ$ $h_{\alpha^{\prime}}$. But this equality follows by the choice of $h_{\alpha^{\prime}}$ and (4). Thus, $h=h_{\alpha^{\prime}}$ is the desired semiconjugacy for any $\alpha^{\prime} \in I$.

An analogous proof can be prepared for the space $\mathbb{F}_{c}^{1}(X)$ of fuzzy numbers on $X$ and for $\mathbb{F}(X)$, respectively.

Theorem 4 For any dynamical system $(X, \varphi)$, there exists a semiconjugacy $h: \mathbb{F}_{c}^{1}(X) \rightarrow \mathbb{K}_{c}(X)$.

Remark 2 For completeness, let us stress ([13]) that in general there is no semiconjugacy between the original dynamical system $(X, \varphi)$ and its extensions $\left(\mathbb{F}^{1}(X), \tilde{\varphi}\right)$ and $(\mathbb{K}(X), \bar{\varphi})$, respectively, and that the same holds for $\left(\mathbb{F}_{c}^{1}(X), \tilde{\varphi}\right)$ and $\left(\mathbb{K}_{c}(X), \bar{\varphi}\right)$.

The last two theorems have several immediate consequences since many dynamical properties are preserved by semiconjugacies. For instance, by using the definition of semiconjugacy, it is an easy exercise to prove Propositions 2-4 and 5-7 and other results of that kind.

We have not mentioned yet whether, for a dynamical system $(X, \varphi)$, any fuzzification $\tilde{\varphi}_{c}$ on $\mathbb{F}_{c}^{1}(X)$ is transitive. There is no general answer to this task, the answer depends on the topological structure of $X$. For instance, if $(X, \varphi)$ is transitive and the space $X$ is totally disconnected (e.g. $\sigma_{n}$ on $\Sigma_{n}$ ) then there exists a bijection (in fact, a conjugacy) $x \mapsto \chi_{\{x\}}$ between $(X, \varphi)$ and $\left(\mathbb{F}_{c}^{1}(X), \tilde{\varphi}_{c}\right)$ and hence the fuzzification is transitive.
On the other hand, it is known (for details and references see e.g. [1]) that certain compact metric spaces (e.g. arcs, intervals, dendrites, continua containing a free arc, irreducible continua of the type $\lambda$ etc.) do not admit a transitive set-valued extension $\bar{\varphi}$ on $\mathbb{K}_{c}(X)$. We immediately obtain from Proposition 5 that no fuzzification $\tilde{\varphi}$ is transitive on the space of fuzzy numbers on such spaces. Consequently, by (1) the fuzzification on such spaces does not admit other common dynamical properties and cannot be exact, strongly mixing, mildly mixing, totally transitive etc. and hence (c.f., exact, totally) Devaney chaotic.

Corollary 1 ([14]) There are dynamical systems $(X, \varphi)$ such that their fuzzifications are not transitive on $\mathbb{F}_{c}^{1}(X)$.

Remark 3 Within the last two subsection we demonstrated (see Corollary 1 and Example 1) that fuzzifications of the simplest one-dimensional dynamical systems (those given by interval maps) does not admit the same dynamical properties as the original dynamical system. Therefore, it doesn't make sense to consider whether various Devaney's chaoses for such fuzzifications.

\subsection{Topological entropy}

However, it has been proved recently [6] that the notion of topological entropy is a reasonable instrument to be considered for fuzzifications on the space of fuzzy numbers. In fact, Theorem 5 is the first result pointing out from the mathematical point of view that the set-valued and fuzzy dynamical systems admit distinct dynamical complexities.

Let us recall some basic notions at first. It is well known that the topological entropy is monotone, i.e.,

$$
A \subseteq B \subseteq X \text { implies } h\left(\left.\varphi\right|_{A}\right) \leq h\left(\left.\varphi\right|_{B}\right)
$$

According to embeddings we mentioned in Lemma 1 we have

$$
h(\varphi) \leq h(\bar{\varphi}) \leq h(\tilde{\varphi})
$$

and

$$
h(\varphi) \leq h\left(\bar{\varphi}_{c}\right) \leq h\left(\tilde{\varphi}_{c}\right)
$$

for any dynamical system $(X, \varphi)$. As it is expected, the inequalities in (7) can be strict as it was shown for set-valued ([11]) and also fuzzy extensions ([6]). In the latter case we proved that very simple dynamics of the original space ensures maximal (i.e., infinite) topological entropy of the fuzzification.

Theorem 5 Let $(X, \varphi)$ be a dynamical system. If $x \in X$ is a point with an orbit $\mathcal{O}_{\varphi}(x)$ containing infinite backward orbit that does not intersect $\omega_{\varphi}(x)$, then the fuzzified map $\Phi$ on $\left(\mathbb{F}_{0}(X), d\right)$, where $d \in$ $\left\{d_{E}, d_{S}, d_{\infty}\right\}$, has infinite topological entropy (i.e., ent $_{d}(\Phi)=\infty$ ). 
Example 2 Let $f \in C(I)$ be defined by $f(x)=x^{2}$. This map provides very simple dynamics on I. This map possesses $\omega$-limit set consisting of just two fixed points 0 and 1 and trajectories of remaining points converge to 0 . Thus $f$ has zero topological entropy but ent $(f)=\infty$ by Theorem 5 .

In the proof of Theorem 5 we used the fact that $\alpha$-cuts of chosen fuzzy sets admits unbounded number of connected components. When only limited number of connected components is admissible, we can obtain much more optimistic results.

In fact, analogous, more optimistic results can be expected for graphs, circler, trees etc. since these compact spaces possess similar structures of the set of $\omega$ limit points. But they cannot be expected in general - it is already known ([1]) that there are spaces (e.g., dendrites) $Y$ and maps $\psi: Y \rightarrow Y$ such that even the setvalued extension has infinite topological entropy, i.e., $\operatorname{ent}\left(\bar{\psi}_{c}\right)=\infty$.

\section{Conclusions}

We contributed to the problem that was established in several papers - e.g. in [12], [9] or [18]. We studied relations among dynamical properties between a given discrete dynamical system and its Zadeh's extension that was considered on the space of fuzzy sets and fuzzy numbers, respectively.

We provided a brief introduction to the problem mentioned above - especially we discussed dynamical properties related to various Devaney chaoses (i.e., the periodic density and transitivity) and we clarified that positive relations between $(X, \varphi)$ and $\left(\mathbb{F}_{c}^{1}(X), \tilde{\varphi}_{c}\right)$ can be hardly expected. Note here that some positive relations are kept in general (see [14] for complete solution).

Additionally, we proved that set-valued extensions of $(X, \varphi)$ are dynamical factors of fuzzy ones. And finally, we clarified that, unlike various Devaney chaoses, the notion of the topological entropy seems to be a reasonable instrument for studying dynamical complexity of fuzzifications defined on the space of fuzzy numbers.

\section{Acknowledgment}

This work was supported the research plan MSM 6198898701 of the Ministry of Education of the Czech Republic.

\section{References}

[1] G. Acosta, A. Illanes, H. Méndez-Lango, The transitivity of induced maps, Topology and its Applications 159 (2009), pp. 1013 - 1033.

[2] L. S. Block and W. A. Coppel, Dynamics in one dimension, Lecture Notes in Mathematics, 1513 Springer-Verlag, Berlin, 1992.

[3] R. Bowen, Entropy for group endomorphisms and homogeneous spaces, Trans. Amer. Math. Soc., 153 (1971), pp. $401-414$.
[4] J. Banks, J. Brooks, G. Cairns, G. Davis, P. Stacey, On Devaney's definition of chaos, Amer. Math. Monthly 99 (1992), pp. 332 - 334.

[5] C. A. Cabrelli, B. Forte, U. M. Molter, E. R. Vrscay, Iterated fuzzy set systems: a new approach to the inverse problem for fractals and other sets, J. Math. Anal. Appl. 171 (1992), pp. 79 - 100.

[6] J. Cánovas, J. Kupka, Topological entropy of fuzzified dynamical systems, Fuzzy Sets and Systems 165 2011, pp. 37-49.

[7] J. Cánovas, J. M. Rodríguez, Topological entropy of maps on the real line, Topology and its Applications 153 (2005), Issue 5-6, pp. 735 - 746.

[8] L. Stefanini, L. Sorini, M. L. Guerra, Simulation of fuzzy dynamical systems using the $L U$ representation of fuzzy numbers, Chaos, Solitons \& Fractals 29 (2006), Is. 3, pp. $638-652$.

[9] P. Diamond, A. Pokrovskii, Chaos, entropy and a generalized extension principle, Fuzzy Sets and Systems 61 (1994), pp. 277 - 283.

[10] E. Glasner, B. Weiss, Sensitive dependence on initial conditions, Nonlinearity 6 (1993), pp. 1067 1075.

[11] J. L. García Guirao, D. Kwietniak, M. Lampart, P. Oprocha, A. Peris, Chaos on Hyperspaces, Nonlinear Analysis: Theory, Methods \& Applications 71, Issue 1-2 (2009), pp. 1 - 8 .

[12] P. E. Kloeden, Compact supported endographs and fuzzy sets, Fuzzy Sets and Systems 4 (1980), pp. 193 $-201$.

[13] J. Kupka, On Fuzzifications of Discrete Dynamical Systems, Information Sciences 181 (2011), 13, pp. 2858-2872.

[14] J. Kupka, On Devaney Chaotic Induced Fuzzy and Set-valued Dynamical Systems, Fuzzy Sets and Systems, doi:10.1016/j.fss.2011.04.006.

[15] J. Kupka, Some chaotic and mixing properties of fuzzified dynamical systems, Information Sciences, submitted.

[16] K. Kuratowski, Topology, Vol. II, Academic Press, London, New York, 1968.

[17] S. M. Pederson, Fuzzy homoclinic orbits and commuting fuzzifications, Fuzzy Sets and Systems 155 (2005), Vol. 3, pp. $361-371$.

[18] H. Román-Flores, Y. Chalco-Cano, Some chaotic properties of Zadeh's extension, Chaos, Solitons and Fractals 35 (2008), pp. 452 - 459.

[19] H. Román-Flores, Y. Chalco-Cano, Robinson's chaos in set-valued discrete systems, Chaos, Solitons and Fractals 25 (2005), pp. 33 - 42.

[20] Y. J. Xue, S. Y. Yang, Synchronization of generalized Henon map by using adaptive fuzzy controller, Chaos, Solitons \& Fractals 17 (2003), pp. 717 - 722.

[21] L. Zadeh, Fuzzy sets, Information and Control 8 (1965), pp. 338-353. 